\title{
Análise comparativa da capacidade funcional de idosos institucionalizados e não institucionalizados
}

Comparative analysis of the functional capacity of institutionalized and non institutionalized elderly

Fisisenectus . Unochapecó Ano 5, n. 1 - Jan/Jun. 2017 p. 3-12

Raíla Oliveira Leão. raila_14@hotmail.com

Fisioterapeuta. Graduada pelo Centro Universitário de Formiga (UNIFOR) - MG.

Natane Moreira de Carvalho. natanecarvalho@gmail.com

Enfermeira. Mestre em Biotecnologia pela Universidade Federal de São João Del Rei (UFSJ). Doutoranda em Patologia pela Universidade Federal de Minas Gerais (UFMG) - MG.

Carolina Marques Carvalho Mitre Chaves. carolinamchaves@uol.com.br Fisioterapeuta. Mestre em Ciências da Reabilitação pela Universidade Federal de Minas Gerais (UFMG). Docente do Curso de Fisioterapia da Universidade de Itaúna (UIT) - MG.

Andrei Pereira Pernambuco.pernambucoap@ymail.com

Fisioterapeuta. Doutor em Biologia Celular pela Universidade Federal de Minas Gerais (UFMG). Docente do Curso de Fisioterapia, Educação Física e Biomedicina do Centro Universitário de Formiga (UNIFOR-MG). Docente do Curso de Fisioterapia da Universidade de Itaúna (UIT) - MG.

\section{Resumo}

Introdução: O Brasil será em 2025, o sexto país do mundo com maior número de idosos. Uma parcela desses continuará participando ativamente da sociedade, enquanto a outra viverá em instituições de longa permanência. Objetivo: Avaliar e comparar a capacidade funcional de idosos institucionalizados (IIN) e idosos comunitários (ICO). Materiais e métodos: Participaram do estudo 20 idosos, de ambos os gêneros, com idade entre 60 e 80 anos, que foram recrutados ou em uma instituição de longa permanência para idosos ou em uma unidade básica de saúde. Os participantes foram divididos em dois grupos com 10 participantes. 0 índice de Katz, a Escala de Lawton e Brody, e o Timed Get Up and Go (TUG) foram utilizados para coleta dos dados. Resultados: No grupo de ICO foram encontradas médias de 0,10 $\pm 0,32$ pontos no Índice de Katz, 16,90 $\pm 3,52$ pontos na Escala de Lawton e Brody e 11,68 $\pm 5,92$ segundos no teste TUG, já no grupo de IIN as médias foram, 0,50 \pm 0,71 pontos, $11,70 \pm 1,89$ pontos e $19,25 \pm 8,52$ segundos, nos respectivos instrumentos. A comparação intergrupos demonstrou que não houve diferenças significativas no que se refere à capacidade de realização de atividades básicas de vida diária $(p=0,1336)$. Contudo ocorreram diferenças significativas a favor do grupo ICO na capacidade de realizar atividades instrumentais de vida diária $(p=0,0006)$ e no teste TUG $(p=0,0089)$. Conclusão: Os achados sugerem que a institucionalização pode acelerar o surgimento e agravamento de incapacidades funcionais.

\section{Palavras-chave}

Funcionalidade; Incapacidade; Envelhecimento; Institucionalização.

\section{Fisißenectus}




\begin{abstract}
Introduction: Brazil will be in 2025, the sixth country in the world with the largest number of elderly. A portion of these will continue to participate actively in society, while the other will live in long-term institutions. Objective: To evaluate and compare the functional capacity of institutionalized elderly (INE) and community elderly (COE). Materials and methods: Twenty elderly people of both genders, 60 to 80 years of age, recruited or in a long-term institution for the elderly or in a basic health unit participated in the study. Participants were divided into two groups with 10 participants. The Katz index, the Lawton and Brody Scale, and the Timed Get Up and Go (TUG) were used for data collection. Results: In the COE group averages of $0.10 \pm 0.32$ points were found in the Katz Index, $16.90 \pm 3.52$ points in the Lawton and Brody Scale, and $11.68 \pm 5.92$ seconds in the TUG test, In the INE group, the means were $0.50 \pm 0.71$ points, $11.70 \pm 1.89$ points and $19.25 \pm 8.52$ seconds, in the respective instruments. The intergroup comparison showed that there were no significant differences regarding the ability to perform basic activities of daily living $(p=0.1366)$. However, there were significant differences in favor of the COE group in the ability to perform instrumental activities of daily living $(p=0.0006)$ and in the TUG test $(p=0.0089)$. Conclusion: The findings suggest that institutionalization can accelerate the onset and worsening of functional disabilities.
\end{abstract}

\title{
Keywords
}

Functionality; Incapacity; Aging; Institutionalization.

\section{Introdução}

$\infty \times \infty \times \infty \times \infty \times \infty \times \infty \times \infty \times \infty \times \infty \times \infty \times \infty \times \infty)$

No Brasil, é considerado idoso qualquer indivíduo com idade igual ou superior a 60 anos. E segundo estimativas, em 2025 o Brasil será o sexto país do mundo em relação ao percentual de idosos em relação à população geral, de acordo com as estimativas a proporção de idosos passaria dos atuais 2,7\% para $14,7 \%^{1}$. Essa realidade não se limita ao Brasil e é encontrada na maioria dos países em desenvolvimento, devido ao fenômeno conhecido como transição demográfica, famílias com menores números de filhos e com ampla expectativa de vida ${ }^{2}$.

0 envelhecimento trata-se de um processo biofisiológico natural que envolve alterações nas estruturas e funções corporais de forma inevitável, gradativa e irreversível ${ }^{3}$. Fatores intrínsecos como os genéticos e fatores extrínsecos, como os ambientais podem influenciar positivamente ou negativamente no processo de envelhecimento. Quando esse acontece de forma saudável, se dá o nome de senescência e é marcado pela preservação das funções cognitivas e funcionais, mas quando ocorre de forma patológica, recebe o nome de senilidade ${ }^{4}$.

Com o crescimento da população idosa, uma parcela importante desses indivíduos passará a viver em instituições de longa permanência, processo conhecido como institucionalização. Esse fenômeno é cada vez mais comum, já que as mudanças na estrutura familiar, o acúmulo de atividades do cotidiano e a necessidade de cuidados específicos aos idosos, contribuem com opção pela institucionalização $0^{5}$. Contudo, o impacto desse fenômeno sobre a saúde e qualidade de vida dos idosos que vivenciam tal situação ainda é pouco compreendido por pesquisadores e profissionais da saúde ${ }^{1}$.

É sabido que os fatores ambientais podem influenciar positivamente ou negativamente a saúde e os aspectos relacionados à saúde dos indivíduos e populações ${ }^{6}$. Além do mais, o conceito de saúde não pode ser definido apenas pela ausência de doenças e, deve considerar também a preservação da capacidade funcional do individuo $0^{7,8}$. Portanto, a saúde plena envolve, dentre outros constructos, a capacidade preservada de se realizar atividades básicas de vida diária (ABVD) e atividades instrumentais de vida diária (AIVD). Ambas determinantes para a atividade e participação social dos indivíduos ${ }^{6,8}$. Diante do exposto, o estudo teve como propósito avaliar e comparar a capacidade funcional de idosos institucionalizados e idosos comunitários a fim de se compreender o impacto da institucionalização sobre a capacidade funcional dessas pessoas. 


\section{Materiais e métodos}

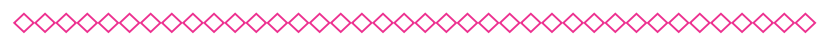

\section{Tipo de estudo}

Estudo observacional, transversal e retrospectivo.

\section{Participantes}

Os participantes foram recrutados por busca ativa em uma unidade básica de saúde e em uma instituição de longa permanência para idosos do município de Iguatama - MG. Para participar do estudo os participantes precisaram cumprir os seguintes critérios de inclusão: possuir idade entre 60 e 80 anos, ser capaz de deambular independentemente de dispositivos de auxílio ou assistência de terceiros, estar cadastrado em uma instituição de longa permanência para idosos ou estar cadastrado no Programa de Saúde da Família do município, possuir estado cognitivo apropriado para a participação no estudo (avaliado pelo Mini Exame do Estado Mental) ${ }^{9}$. Os seguintes critérios de exclusão foram utilizados: portadores de doenças inflamatórias crônicas, autoimunes e ou neurológicas, idosos temporariamente ou permanentemente acamados, nível cognitivo não condizente com a participação no estudo, déficit importante da acuidade visual e ou auditiva, não assinatura do termo de consentimento livre esclarecido (TCLE).

A busca ativa por participantes resultou em 30 potenciais voluntários, sendo 15 provenientes da UBS e 15 provenientes da instituição de longa permanência. Após a aplicação dos critérios de exclusão a amostra final do estudo foi definida em 20 idosos, sendo que 10 foram recrutados na UBS e inscritos no grupo de idosos comunitários (ICO) e 10 na instituição de longa permanência, que foram inscritos no grupo de idosos institucionalizados (IIN).

\section{Instrumentos de coleta de dados}

Para avaliação das funções cognitivas, foi utilizado o Mini-Exame do Estado Mental (MEEM). Proposto por Foistein e McHugh em 1975, foi validado no Brasil por Bertolucci et al. (1994) ${ }^{9}$, esse instrumento é composto por questões agrupadas em sete categorias: orientação temporal (cinco pontos), orientação espacial (cinco pontos), memória imediata (três pontos), atenção e cálculo (três pontos), evocação (três pontos), linguagem (oito pontos) e praxia construcional (três pontos), totalizando 30 pontos; a interpretação varia de acordo com o nível de escolaridade do indivíduo. 0 escore predito para um indivíduo analfabeto é $>14$, semianalfabeto o esperado é $>18$, e escolarizado é $>24$. No estudo foram excluídos os indivíduos com pontuação igual ou inferior a 13 pontos.

Para a avaliação da capacidade em realizar as $A B V D$, foi utilizado o Índice de Katz. Esse instrumento foi desenvolvido para avaliar as atividades de vida diária. Os itens são relacionados e organizados para mensurar o grau de dependência ou independência em seis funções básicas: banhar-se, vestir-se, usar o banheiro, transferir-se, ter continência, e alimentar-se. Katz e Akpom (1976) ${ }^{10}$ apresentaram uma versão modificada da escala original, a classificação do Índice se dá pelo número de funções nas quais o indivíduo avaliado é dependente, sendo os índices de zero a seis: zero - independente em todas as seis funções; um - independente em cinco funções e dependente em uma função; dois - independente em quatro funções e dependente em duas; três - independente em três funções e dependente em três; quatro independente em duas funções e dependente em quatro; cinco - independente em uma função e dependente em cinco funções e seis - dependente em todas as seis funções. Sendo assim, quanto maior a pontuação, maior o nível de dependência do indivíduo. Esse instrumento possui validade e confiabilidade testadas ${ }^{7}$.

Para a avaliação da capacidade de se realizar as AIVD foi utilizado o questionário de Lawton e Brody. Esse contempla atividades como: preparar refeições, realizar compras, utilizar transporte, cuidar da casa, utilizar telefone, administrar as próprias finanças, administrar medicamentos. A escala foi desenvolvida por Lawton e Brody e adaptada ao contexto brasileiro por Santos e Júnior em $2008^{11}$. Os idosos são classificados de acordo com a pontuação obtida, ao final são classificados de acordo com a seguinte escala: dependência total $<5$; dependência parcial $>5$ e $<21$, e independência $=21$, A esses valores o escore nesta escala varia entre 7 a 21 pontos $^{12}$. No presente estudo essa escala foi utilizada também para os participantes institucionalizados. Nesse caso a resposta deveria levar em consideração uma 
situação hipotética na qual o participante deveria dizer se sentia apto a realizar a atividade caso fosse necessário ${ }^{11}$.

A capacidade funcional, referente a locomoção foi avaliada por meio do teste Timed Get Up and Go (TUG). Trata-se de um teste funcional simples, proposto por Mathias, Nayak e Isaacs em 1986. 0 paciente é solicitado a levantar-se de uma cadeira (altura do assento de $45 \mathrm{~cm}$ e dos braços de 65 $\mathrm{cm})$, deambular três metros, retornar e sentar-se novamente, enquanto o tempo despendido na realização desta tarefa é cronometrado. Indivíduos independentes sem alterações no equilíbrio realizam o teste em 10 segundos ou menos; com independência em transferências básicas gastam 20 segundos ou menos. Já os indivíduos que necessitam de mais de 30 segundos para realizar o teste são dependentes em muitas atividades de vida diária e na mobilidade ${ }^{13}$.

\section{Coleta dos dados}

Os componentes do grupo de idosos institucionalizados (IIN) foram avaliados dentro da própria instituição, e os idosos que compuseram grupo de idosos comunitários (ICO) foram avaliados em seu respectivo domicilio. Todos os dados coletados foram obtidos em um único momento, por um avaliador experiente, treinado e cegado para os objetivos do estudo.

\section{Análise estatística}

Os dados foram avaliados primeiramente de acordo com a estatística descritiva. Medidas de tendência central (média) e de dispersão (desvio padrão) foram utilizadas. Em sequência foi utilizado o teste de Shapiro-Wilk para avaliar a normalidade dos dados. Para análise intergrupos utilizou-se o Teste $T$ Independente em caso de dados com distribuição normal e, para dados de distribuição anormal foi utilizado o teste de Mann-Whitney. Todos os testes foram realizados no Software GraphPad Prism v.5.0, adotando o nível de significância de $5 \%(p<0,05)$.

\section{Cuidados éticos}

0 protocolo de pesquisa foi iniciado após a aprovação pelo Comitê de Ética em Pesquisas em Humanos do Centro Universitário de Formiga MG, sob o parecer de número 787.757. Todos os participantes assinaram um termo de consentimento livre e esclarecido (TCLE) em duas vias. Esse e demais cuidados éticos foram pautados na resolução 466/12 do Conselho Nacional de Saúde do Ministério da Saúde (CNS/MS).

\section{Resultados}

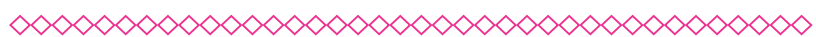

A amostra final do estudo foi composta por 20 participantes, 10 no grupo de idosos comunitários (ICO) e 10 no grupo de idosos institucionalizados (IIN). O grupo ICO foi composto por cinco homens e cinco mulheres, enquanto que o grupo IIN foi composto por quatro homens e seis mulheres. A idade máxima dos participantes no grupo ICO foi de 80 anos, assim como no grupo IIN. No grupo ICO a média de idade foi de 73,40 $\pm 6,72$ anos e no grupo IIN foi de 70,70 \pm 7,98 anos (Tabela 1 e Tabela 2).

Nos instrumentos de coleta de dados, o grupo ICO obteve média de $0,10 \pm 0,32$ pontos no Índice de Katz, 16,9 $\pm 3,51$ pontos na Escala de Lawton e Brody e 11,6 \pm 5,93 segundos no teste TUG. Dados demonstrados na Tabela 1.

Já os indivíduos do grupo IIN obtiveram pontuação média de $0,5 \pm 0,71$ pontos no Índice de Katz, $11,7 \pm 1,89$ pontos na Escala de Lawton e Brody e completaram o teste TUG com média de 19,25 \pm 8,52 segundos. Dados expressos na Tabela 2 .

A comparação intergrupos demonstrou que não houve qualquer diferença significativa entre os grupos no que se refere à idade $(p=0,42)$ (Figura 1 - A) e pontuação no índice de Katz ( $p$ = 0,1336) (Figura $1-B$ ). Contudo, as análises demonstraram haver diferenças significativas à favor do grupo ICO em relação ao grupo IIN, tanto na pontuação na Escala de Lawton e Brody $(p=0,0006)$ (Figura $1-C)$ quanto no Teste TUG $(p=0,0089)$ (Figura $1-D)$.

\section{Discussão}

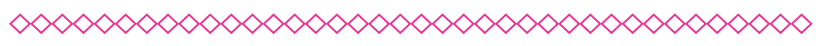

Os resultados do presente estudo demostram, em suma, que idosos institucionalizados apresentam menor capacidade funcional do que idosos 
comunitários, quando avaliados pela Escala de Lawton e Brody e pelo Teste TUG. A idade dos participantes poderia justificar essa diferença, contudo os grupos do estudo foram pareados em relação à idade a fim de se evitar viés de interpretação. Além do mais, se for considerada apenas a média de idade dos grupos, a do grupo ICO é superior à encontrada no grupo IIN. Em relação ao gênero dos participantes, é importante ressaltar que no grupo de ICO o percentual de mulheres na amostra foi minimamente superior ao do grupo IIN. É sabido que as mulheres possuem menos força muscular que os homens ${ }^{14}$. Contudo apenas esse fato não explicaria os déficits na realização de atividades instrumentais de vida diária nem no desempenho no Teste TUG, já que tais testes não demandam força física e sim habilidades motoras e agilidade. A população feminina do Brasil vem aumentando mais do que a masculina, e esse fenômeno é explicado, pelo menos em parte pelo aumento da expectativa de vida das mulheres que adotam maiores cuidados na prevenção e promoção da saúde do que os homens ${ }^{15}$.

Na pontuação relacionada ao Índice de Katz, que avalia, sobretudo as atividades básicas de vida diária, nenhuma diferença significativa foi encontrada entre os dois grupos. Os dados do presente estudo mostram que os participantes do estudo apresentam capacidade preservada para a realização de atividades básicas de vida diária. Esses dados corroboram os achados de Costa, Nakatani e Bachion (2006) ${ }^{16}$ e de Gonçalves et al. (2010) ${ }^{17}$, mas, por outro lado, contradizem os de Oliveira et al. (2009) ${ }^{18}$ que encontrou um percentual de $54 \%$ de idosos com dependência para realizar atividades básicas. A contradição entre os estudos apresentados pode ser justificada pelo fato de que Oliveira et al. (2009) ${ }^{18}$ trabalhou com uma amostra com média de idade de aproximadamente 78 anos e no presente estudo, bem como no de Costa, Nakatani e Bachion (2006) ${ }^{16}$ e de Gonçalves et al. $(2010)^{17}$ a média de idade dos participantes era inferior. A partir de 80 anos, mesmo os idosos que apresentam um envelhecimento saudável, tendem a apresentar um decréscimo fisiológico na capacidade de realizar as tarefas do dia-a-dia ${ }^{16}$. Neste estudo, apesar de os idosos institucionalizados apresentarem mais dificuldade durante a realização de ABVD, esta dificuldade não compromete a rotina diária, já que são capazes de realizar a maioria das atividades sem qualquer auxílio, assim como fazem os idosos comunitários. Já foi descrito que a intensidade e a frequência de como ocorre o comprometimento na realização das atividades está relacionado com as condições de saúde, a longevidade, e o estilo de vida e ao ambiente em que se vive ${ }^{3,16}$.

Diferentemente da pontuação no Índice de Katz, na Escala de Lawton e Brody foi possível identificar diferenças significativas entre os dois grupos do estudo. Nessa escala que avalia atividades instrumentais de vida diária, os participantes do grupo IIN apresentaram pior desempenho do que os indivíduos do grupo ICO, dados que corroboram os achados de outro estudo ${ }^{19}$. No presente estudo, no grupo ICO 30\% dos idosos são independentes e $70 \%$ parcialmente dependentes, resultados também semelhantes aos de outro estudo ${ }^{16}$. Já no grupo IIN $100 \%$ dos indivíduos são parcialmente dependentes, achados semelhantes foram publicados por Lacerda et al. (2010) ${ }^{12}$ que identificaram um percentual de $90 \%$ de idosos parcialmente dependentes vivendo em uma instituição de longa permanência para idosos. É importante frisar que no grupo ICO os participantes que são parcialmente dependentes, o são em ações como: realizar compras, utilizar medicamentos e viajar. Os achados apresentados aqui podem sugerir que o ambiente habitado pelos idosos institucionalizados possa estar atuando como barreira para a execução das atividades, já que os idosos institucionalizados possuem menos autonomia para realizar suas atividades rotineiras ${ }^{20}$. Há ainda que se mencionar, que o processo de envelhecimento, naturalmente conduz a uma redução da capacidade ou desempenho nas atividades, mas que, de acordo com os dados aqui apresentados a institucionalização pode acelerar esse processo. Isso ocorre, pelo menos em parte, pelo fato de que pessoas institucionalizadas encontram-se mais restritas socialmente e apresentam limitações nas atividades, pois grande parte das atividades que lhe cabiam, acabam sendo realizadas por terceiros, acelerando assim o processo de dependência ${ }^{12,19}$.

No teste Timed Get Up and Go (TUG), situação semelhante foi observada, já que os participantes do grupo de IIN realizaram o teste com média de tempo significativamente superior aos participantes 
do grupo ICO. Os resultados de outro estudo ${ }^{21}$ corroboram os achados aqui apresentados. No presente estudo, $60 \%$ dos idosos poderiam ser considerados independentes, $30 \%$ independentes em transferências básicas e 10\% dependentes em atividades do cotidiano e da mobilidade. Já no grupo IIN nenhum indivíduo foi considerado independente, $60 \%$ são independentes em transferências básicas e $40 \%$ podem ser considerados dependentes em atividades do cotidiano e da mobilidade. A capacidade de se transferir, e a mobilidade são atividades de grande importância na vida cotidiana do indivíduo e a manutenção destas habilidades é importante para a independência e efetiva realização das atividades de vida diária, sejam essas básicas ou instrumentais ${ }^{21}$. A redução ou até mesmo a perda dessas habilidades pode causar prejuízos funcionais aos idosos. A redução da mobilidade pode ser observada em ambos os grupos, mas é mais evidenciada em idosos que residem em instituições de longa permanência ${ }^{21}$. É provável que idosos comunitários permaneçam ativos por mais tempo, e por isso, usufruem de sua independência por períodos mais duradouros. Vários são os fatores que podem contribuir para esta realidade, mas os fatores contextuais, sobretudo, os ambientais devem ser ressaltados, tais como, o apoio e de amigos e familiares e a demanda causada pela participação social ${ }^{6}$. Além disso, o tempo de institucionalização pode influenciar negativamente na redução da funcionalidade, por resultar em mais tempo de isolamento social, alterações psicológicas, redução da atividade física e perda da autonomia. Como já descrito, idosos institucionalizados são mais frágeis emocionalmente e fisicamente ${ }^{22}$.

0 processo de institucionalização também apresenta pontos positivos e não é objetivo desse estudo desencorajar idosos e familiares a optarem por essa alternativa. Contudo, o modelo atual pode ser repensado a fim de se estimular a atividade e a autonomia das pessoas institucionalizadas, seja em grupo ou individualmente. É importante mencionar que a opção pela institucionalização se dá, sobretudo quando o idoso demanda cuidados de saúde e ou quando a família não possui tempo suficiente para fornecer os cuidados necessários ao bem estar do idoso ${ }^{23,24}$. Sendo assim, a inserção de fisioterapeutas, terapeutas ocupacionais, psicopedagogos, educadores físicos dentre outros profissionais capazes de contribuir com o bem estar do indivíduo institucionalizado.

As principais limitações relacionadas ao estudo foram: A) Tamanho amostral limitado, contudo os achados do presente estudo, quase sempre corroboraram os resultados de estudos que utilizaram grandes amostras. B) Desenho do estudo, transversal e retrospectivo, é sabido que estudos com desenho longitudinal e prospectivos fornecem evidências mais robustas, além de permitir o acompanhamento da situação dos sujeitos ao longo do tempo. C) Análises de correlação ou regressão não foram utilizadas a fim de se tentar sugerir quais variáveis mais interferiram na perda da capacidade funcional. D) Dificuldade de se inserir no estudo idosos que não apresentavam qualquer doença associada, dada a faixa etária dos participantes.

\section{Conclusão}

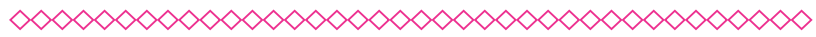

Os resultados do presente estudo sugerem que a capacidade funcional de idosos institucionalizados encontra-se reduzida quando comparada com a de idosos não institucionalizados. Os achados do presente estudo sugerem que isso é devido aos fatores ambientais relacionados à institucionalização. Deste modo, a redução da funcionalidade em idosos institucionalizados deve ser alvo de preocupação por parte de profissionais da saúde, pesquisadores, gestores públicos e de responsáveis pelas instituições de longa permanência. A fim de se minimizar tais problemas intervenções multidisciplinares precisam ser prontamente repensadas e implementadas a fim de se prevenir e promover a saúde dessa população.

\section{Referências}

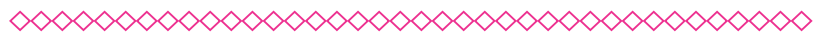

1. Silva SLA, Vieira RA, Arantes P, Dias RC. Avaliação de fragilidade, funcionalidade e medo de cair em idosos atendidos em um serviço ambulatorial de Geriatria e Gerontologia. Fisioterapia e Pesquisa 2009;16(2).

2. Rigotti JIR. Transição demográfica. Educação \& Realidade 2012;37(2). 
3. Schneider RH, Marcolin D, Dalacorte RR. Avaliação funcional de idosos. Scientia Medica 2008;18(1).

4. Smanioto FN, Haddad MCF. Índice de Katz aplicado a idosos institucionalizados. Revista Rene 2011;12(1):18-23.

5. Soares E, Coelho MO, Carvalho, SMR. Capacidade funcional, declínio cognitivo e depressão em idosos institucionalizados: possibilidade de relações e correlações. Revista Kairós Gerontologia 2012;15(5):117-139.

6. Organização Mundial da Saúde (OMS). CIF: Classificação Internacional de Funcionalidade, Incapacidade e Saúde. $1^{\text {a }}$ ed. São Paulo-SP: EDUSP; 2003.

7. Duarte $Y A O$, Andrade CL, Lebrao ML. O Índex de Katz na avaliação da funcionalidade dos idosos. Revista da Escola de Enfermagem - USP 2007;2(4):317-25.

8. Maciel MG. Atividade física e funcionalidade do idoso. Motriz 2010;16(4).

9. Bertolucci PHF, Brucki SMD, Campacci SR, Juliano Y. O Mini-Exame do Estado Mental em uma população geral: impacto da escolaridade. Arq. Neuro-Psiquiatria 1994;52(1):01-07.

10. Katz S, Akpom CA. A measure of primary sociobiological functions. Int J Health Serv 1976;3(6):493-508.

11. Santos RL, Júnior JSV. Confiabilidade da versão brasileira da escala de atividades instrumentais da vida diária. Revista Brasileira de Promoção de Saúde 2008;21(4):290-6.

12. Lacerda JA, Moreira LD, Souza LLC, Santos EV, Araújo TLM, Bruno RX. Capacidade de Idosos Institucionalizados para Realizar Atividades Instrumentais de Vida Diária. Revista Inspirar 2010;2(2).

13. Nunciato $A C$, Pereira BC, Silva AB. Métodos de avaliação da capacidade física e qualidade de vida dos idosos: revisão de literatura. Saúde Rev 2012;12(32):41-48.
14. Raso V, Matsudo SMM, Matsudo VKR. A força muscular de mulheres idosas decresce principalmente após oito semanas de interrupção de um programa de exercícios com pesos livres. Rev Bras Med Esporte 2001;7(6):177-86.

15. Kuchemann BA. Envelhecimento populacional, cuidado e cidadania: velhos dilemas e novos desafios. Soc Estado 2012;27(1).

16. Costa EC, Nakatani AYK, Bachion MM. Capacidade de idosos da comunidade para desenvolver Atividades de Vida Diária e Atividades Instrumentais de Vida Diária. Acta Paul Enferm 2006;19(1):43-48.

17. Gonçalves LHT, Silva AH, Mazo GZ, Benedetti TRB, SANTOS SMA, MARQUES S, et al. 0 idoso institucionalizado: avaliação da capacidade funcional e aptidão física. Cad Saúde Pública 2010;9(26).

18. Oliveira MARG, Miranda CMV, Melo ECF, Miranda MMV, Faustino MCB. Avaliação da capacidade de realização das atividades básicas de vida diária em idosos institucionalizados de Santos-SP, através do Índice de Barthel. Revista Unilus Ensino e Pesquisa 2009;6(11).

19. Araújo MOPH, Ceolim MF. Avaliação do grau de independência de idosos residentes em instituições de longa permanência. Rev Esc Enferm USP 2007;3(41):378-385.

20. Nunes MCR, Ribeiro RCL, Rosado LEFPL, Franceschini SC. Influência das características sociodemográficas e epidemiológicas na capacidade funcional de residentes em Ubá, Minas Gerais. Revista Brasileira de Fisioterapia 2009;13(5):376-82.

21. Begate PSI, Ricardo ACMI, Sawazki G. Avaliação do desempenho funcional de idosos institucionalizados e não Institucionalizados através do teste de mobilidade timed up and go (TUG). Revista Funcional 2009;2(2):43-52.

22. Rodini C, Ferreira LTD, Pirré GE, Hino M, Alfieri FM, Riberto $M$, et al. Estudo comparativo entre a Escala de Equilíbrio de Berg, o Teste 
Timed Up \& Go e o Índice de Marcha Dinâmico quando aplicadas em idosos hígidos. Acta Fisiátr 2008;15(4):267-268.

23. Leite MAG. Gestão da qualidade de vida e da dependência em idosos institucionalizados nas organizações do terceiro setor. Dissertação [Mestrado] - Vila Real; 2011.
24. Davim RMB, Torres GV, Dantas SMM, Lima, VM. Estudo com idosos de instituições asilares no município de Natal-RN: características socioeconômicas e de saúde. Rev LatinoAmericana Enfermagem 2004;3(12):518-524. 


\section{Anexos}

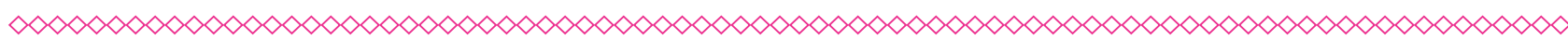

Tabela 1 - Caracterização da amostra e resultados individuais obtidos nos instrumentos de coleta de dados em indivíduos do grupo ICO.

$\begin{array}{lccccc}\text { Indivíduo } & \text { Gênero } & \text { Idade } & \text { Índice de Katz } & \text { Escala Lawton e Brody } & \text { Teste: TUG } \\ 1 & \text { M } & 80 & 0 & 15 & 12,54 \\ 2 & \mathrm{~F} & 64 & 0 & 21 & 7,63 \\ 3 & \mathrm{M} & 71 & 0 & 20 & 7,45 \\ 4 & \mathrm{M} & 80 & 0 & 13 & 15,27 \\ 5 & \mathrm{~F} & 76 & 0 & 20 & 8,74 \\ 6 & \mathrm{~F} & 80 & 0 & 17 & 9,25 \\ 7 & \mathrm{~F} & 74 & 0 & 16 & 8,58 \\ 8 & \mathrm{M} & 64 & 1 & 11 & 10,58 \\ 9 & \mathrm{~F} & 79 & 0 & 15 & 27,13 \\ 10 & \mathrm{M} & 66 & 0 & 21 & 9,59 \\ \text { Média } & & \mathbf{7 3 , 4 0} & \mathbf{0 , 1 0} & \mathbf{1 6 , 9 0} & \mathbf{1 1 , 6 8} \\ \text { Desvio Padrão } & & \mathbf{6 , 7 2} & \mathbf{0 , 3 2} & \mathbf{3 , 5 1} & \mathbf{5 , 9 3}\end{array}$

Resultados individuais dos componentes do grupo de idosos comunitários (ICO).

(clique para voltar ao texto)

Tabela 2 - Caracterização da amostra e resultados individuais obtidos nos instrumentos de coleta de dados em indivíduos do grupo IIN.

\begin{tabular}{lccccc} 
Indivíduo & Gênero & Idade & Índice de Katz & Escala Lawton e Brody & Teste: TUG \\
1 & $\mathrm{~F}$ & 71 & 0 & 14 & 19,15 \\
2 & $\mathrm{~F}$ & 75 & 0 & 12 & 14,15 \\
3 & $\mathrm{M}$ & 62 & 0 & 11 & 13,74 \\
4 & $\mathrm{~F}$ & 80 & 1 & 10 & 30,45 \\
5 & $\mathrm{~F}$ & 80 & 2 & 9 & 36,12 \\
6 & $\mathrm{~F}$ & 80 & 0 & 15 & 20,15 \\
7 & $\mathrm{M}$ & 64 & 1 & 10 & 23,19 \\
8 & $\mathrm{M}$ & 60 & 0 & 13 & 10,23 \\
9 & $\mathrm{M}$ & 63 & 1 & 11 & 11,51 \\
10 & $\mathrm{~F}$ & $\mathbf{7 2}$ & 0 & 12 & $\mathbf{1 3 , 7 9}$ \\
Média & & $\mathbf{7 0 , 7 0}$ & $\mathbf{0 , 5 0}$ & $\mathbf{1 1 , 7 0}$ & $\mathbf{1 9 , 2 5}$ \\
Desvio Padrão & & $\mathbf{7 , 9 9}$ & $\mathbf{0 , 7 1}$ & $\mathbf{1 , 8 9}$ & $\mathbf{8 , 5 2}$ \\
\hline
\end{tabular}

Resultados individuais dos componentes do grupo de idosos institucionalizados (IIN). 
Figura 1 - Resultados obtidos pelos grupos ICO e INN em cada uma das variáveis de interesse
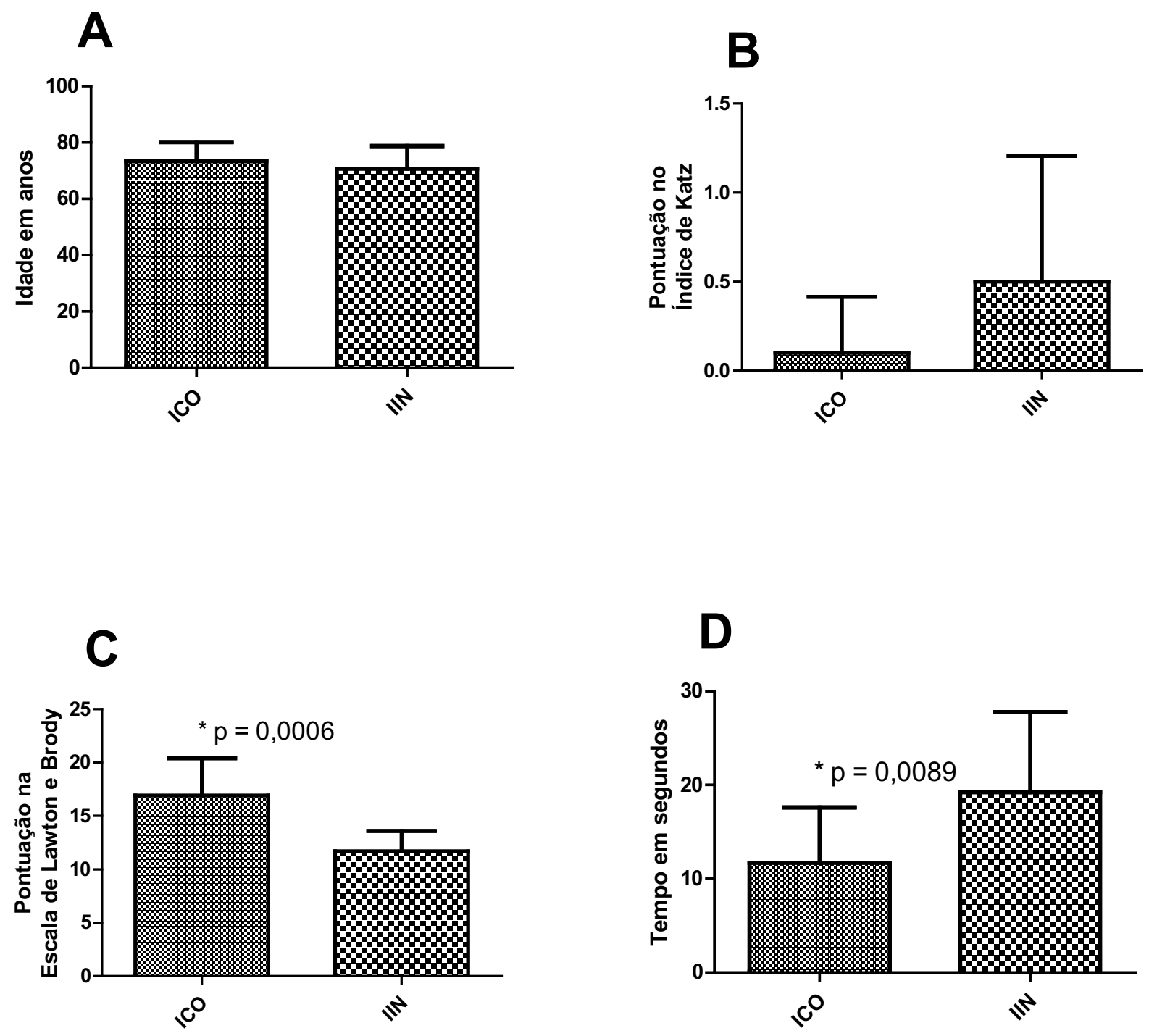

Os gráficos representam a pontuação média e o desvio padrão dos grupos de idosos comunitários (ICO) e de idosos institucionalizados (IIN) nas variáveis de interesse do estudo: A) Idade; B) Índice de Katz; C) Escala de Lawton e Brody; D) Timed Get Up and Go (TUG). Os asteriscos representam as diferenças significativas entre os grupos com valor de $p \leq 0,05$.

(clique para voltar ao texto) 